\title{
Stage I Uterine Corpus Leiomyosarcoma AJCC v8
}

National Cancer Institute

\section{Source}

National Cancer Institute. Stage I Uterine Corpus Leiomyosarcoma A/CC v8. NCI

Thesaurus. Code C139871.

Stage I includes: T1, N0, M0. T1: Uterine corpus leiomyosarcoma with tumor limited to the uterus. N0: No regional lymph node metastasis. M0: No distant metastasis. (AJCC 8th Ed.) 